\title{
LE COPERTURE TOMBALI MUSIVE NELLA TARRACONENSIS NEL SECOLI IV E V D.C.: ISPIRAZIONI E BOTTEGHE
}

Tomb mosaics of Tarraconensis of IV-V centuries: inspirations and workshops

LUIGI QuATTROCCHI

Instituto de Cultura y Tecnología UC3M

Recibido: 16/02/2016

Revisado: 10/03/2017
Aceptado: $19 / 05 / 2017$

Publicado: 23/06/2017

\section{RiASSUNTO}

L'articolo è incentrato sullo studio di 15 coperture funerarie ritrovate nella Tarraconensis. Si fornisce inizialmente un quadro sintetico di tutti i mosaici presi in esame, per poi cercare di individuare quali potessero essere, in primo luogo, le ispirazioni per la realizzazione di queste coperture. In seguito si studiano le ipotetiche botteghe che misero in posa i mosaici, studio che porta alla scoperta di due sicure botteghe, operanti l'una nell'attuale costa catalana, e un'altra nella provincia di Huesca.

\section{RESUMEN}

El articulo está basado sobre el estudio de 15 cubiertas funerarias encontradas en la Tarraconensis. Ademas de un cuadro de sintesis de cada mosaico, se busca de analizar cuál es la fuente ispiradoras de los mismos. En seguida se estudian los hipoteticos talleres que realizaron estos mosaicos, un estudio que nos permite adfirmar la presencia de dos seguros talleres, uno que trabajó en la costa de Cataluña u otro en la provincia de Huesca.

\section{Parole chlave}

Mosaici funerari; sepolture; Cristianesimo; mosaici paleocristiani; Hispania.

\section{Palabras clave}

Mosaicos funerarios; sepulturas; Cristianismo; mosaicos paleocristianos; Hispania.

1.quattrocchi@hotmail.it 


\section{INTRODUZIONE}

Le coperture tombali musive, chiamate anche mosaici funerari, ebbero la loro origine e maggiore fioritura in territorio nord africano. Nati sul finire del III secolo d.C. videro nel IV e soprattutto nel V, un notevole successo, tanto che vennero esportato in quasi tutto il Mediterraneo occidentale. Sembrerebbero prodotti destinati alla comunità cristiana, considerando che la quasi totalità degli esemplari hanno una forte connotazione religiosa, con simboli o frasari tipici dei cristiani dei primi secoli. Questa esportazione toccò anche la storica regione della Tarraconensis, la zona dell'Hispania che possiede il maggior numero di esemplari ritrovati. In totale si hanno 15 mosaici $^{1}$ superstiti in questa regione, sebbene in passato il loro numero dovesse essere maggiore. Individuare le botteghe risulta estremamente difficile, sebbene si possano portare avanti delle ipotesi.

\section{LE COPERTURE MUSIVE: QUADRO SINTETICO}

Come accennato, sono giunte sino a noi 15 coperture musive ${ }^{2}$, dislocate in diversi punti della Tarraconensis (fig. 1), le quali sono suddivisibili in base alla loro tipologia e alla loro composizione all'interno del campo musivo. Così facendo si avranno quattro gruppi di mosaici del tipo: mo-



Fig. 1 Distribuzione delle coperture tombali musive nella Tarraconensis (elaborazione dell'Autore)

1 Tutti i mosaici presenti in questo articolo sono tessellati.

2 I pavimenti sono di più, ma in questa sede non verranno considerati i mosaici dei quali si ha solo la notizia della scoperta, e dei quali non risulta esistente nessuna documentazione fotografica nopartito, bipartito, tripartito e quadripartito, con una netta prevalenza della tipologia bipartita (fig. 2)

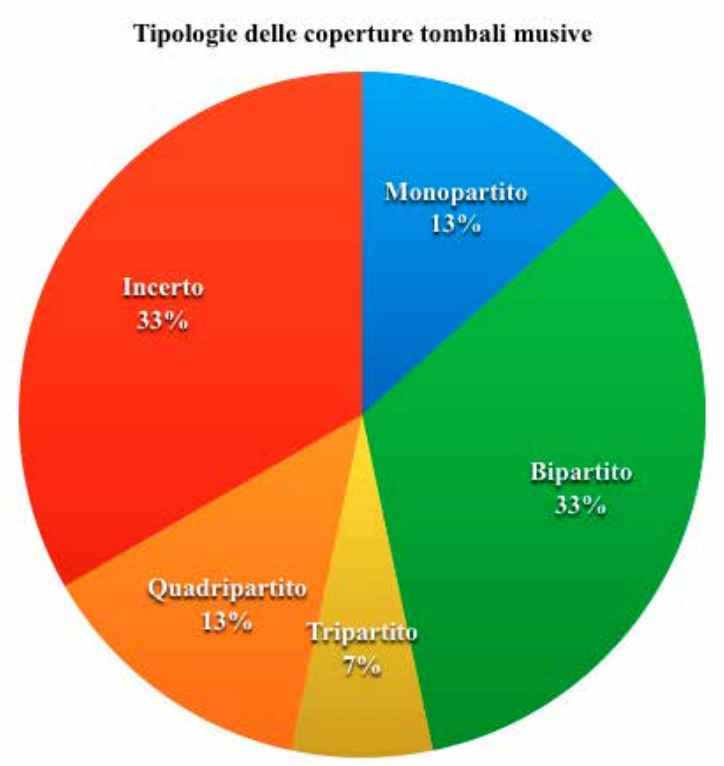

Fig. 2 Tipologie di coperture tombali musive nella Tarraconensis (elaborazione dell'Autore)

Del gruppo monopartito afferiscono due mosaici:

1.

Mosaico ritrovato a Barcellona nella Piazza Antonio Maure e ora conservato presso il Museo de Historia de la Ciudad di Barcellona (fig. 3). Misura 211 x $64 \mathrm{~cm}$, presenta tessere bianche, nere, arancioni, rosse e verdi, con decorazione vegetale e chrismon, e cornice decorata da una treccia a doppio capo, databile al primo quarto del $\mathrm{V}$ secolo ${ }^{3}$. In ottimo stato di conservazione (Balil, 1955, 142; De Palol, 1967,

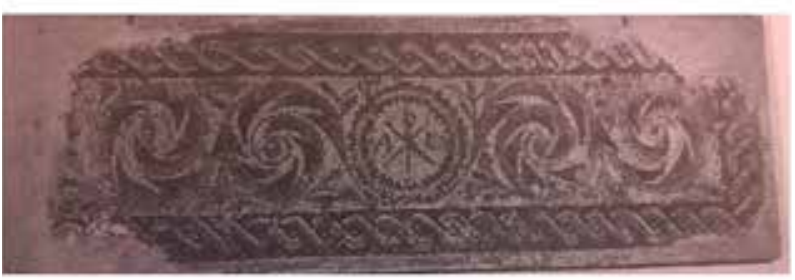

Fig. 3 Mosaico n. 1 ritrovato a Barcellona, Museo de Historia de la Ciudad di Barcellona (da Gómez Pallarès 2002)

3 In mancanza dei dati di scavi le datazioni ivi fornite si riferiscono ad analisi stilistiche e paleografiche; l'unica eccezione si ha per il mosaico funerario 9, sotto il quale furono rinvenute tre monete che ci offrono un terminus ante quem. 
326; Schlunk y Hauschild, 1978, 23; Gómez Pallarès y Mayer, 1996, 54; Gómez Pallarès, 2002, 31-32).

\section{2.}

Mosaico rinvenuto nella Necropoli de Francolí a Tarragona e ora conservato al Museo Nacional Arqueológico de Tarragona, con numero di inventario 2648 (fig. 4). Misura 87 x $52 \mathrm{~cm}$, con tessere di colore bianco, nero, blu, giallo, ocra, grigio, rosso e verde. La cornice è resa da una treccia a due capi e il campo musivo è decorato dalla raffigurazione del defunto nelle vesti del Buon Pastore, una raffigurazione idealizzata, che prevede un giovane imberbe che veste una corta tuni$\mathrm{ca}$, in posizione stante, col braccio destro alzato e il braccio sinistro parallelo al corpo. Completano la raffigurazione due colombe posizione sopra il capo della figura e un chrismon. Il mosaico si data al V secolo. Lacunoso $\mathrm{ma}$ in discreto stato di conservazione (De Palol, 1967, 329; Del Amo, 1979, 107,101; Gómez Pallarès y Mayer, 1996, 77; De Palol, 1999, 303, Gómez Pallarès, 2002, 69-70).

Il gruppo bipartito è composto da:

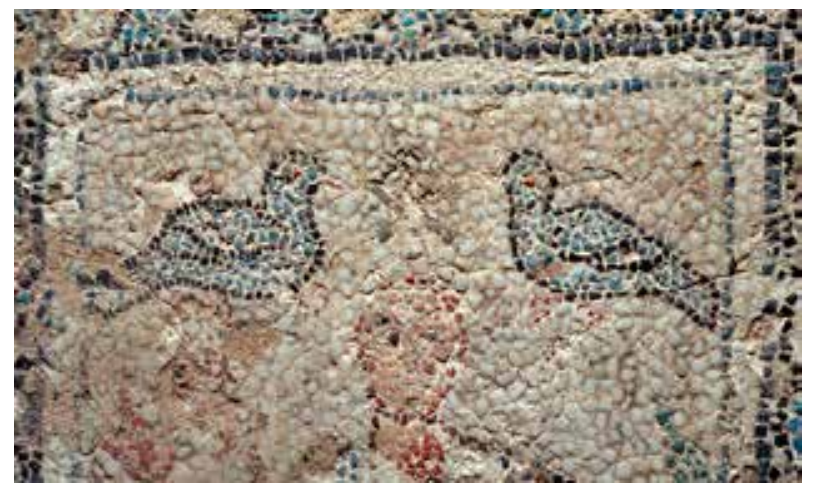

Fig. 4 Mosaico n. 2 ritrovato nella Necropoli de Francolí, Tarragona, Museo Nacional Arqueológico de Tarragona, n. Inv. 2648 (foto dell'Autore)

3.

Il mosaico è stato ritrovato a Terrassa e oggi si trova in situ nella Iglesias de Santa Maria. Misura $34 \times 25 \mathrm{~cm}$, con tessere bianche e nere e recante l'iscrizione:

\section{SECURUS [---]/ CECIL [---]/ N+[---]/ [------] / ES [---] / EST E [---].}

Si è ipotizzato che sia stato riutilizzato e che in origine appartenesse a una copertura della fine del IV secolo. Molto lacunoso, in pessimo stato di conservazione (Puig i Cadafalch, 1947, 539-540; De Palol, 1967, 327; Barral, 1978, n. 145; Álvarez y Mayer - Rodà, 1987, 546-547; Gómez, Pallarès y Mayer, 1996, 55; Gómez Pallarès, 2002, 32).

4.

Mosaico scoperto a Coscojuela de Fantova (Del Arco 1919, 127-142) e oggi conservato presso il Museo de Huesca (fig. 5). Misura 230 x 84 cm, con tessere di colore bianco, grigio, rosso, rosa, e tessere in pasta vitrea dei colori blu e verde. Presenta l'iscrizione:

MACEDONIO PR B/ C (hedera) B CONIVGI (hedera)/ SUO (hedera) MARIA (hedera)/ [-----] VM (hedera)/ AD[------].

La decorazione è data da un bordo con treccia a doppio capo, e nel campo musivo la figura del Buon Pastore, che veste una corta tunica e con le mani nel petto, intente a tenere le zampe di un agnello. Lacunoso ma in buono stato di conservazione. Databile alla seconda metà del IV secolo (Del Arco, 1921; De Palol, 1967, 331-332; Schlunk y Hauschild, 1978, 22-23; Lostal, 1980, 41; Domínguez, Magallón y Casado, 1984, 77-78; Fernández Galliano, 1987, n. 106; Beltrán Lloris, 1987, n. 8; Gómez Pallarès y Mayer, 1996, 58; De Palol, 1999, 303, Gómez Pallarès, 2002, 35-36; Beltrán Lloris, 2004, 49-51; Blázquez Martínez, 2014, 253).

\section{5.}

Come il mosaico precedente, anche questo fu scoperto nel complesso di Coscojuela de Fantova, e risulta conservato nel Museo de Huesca (fig. 6). Misura $225 \times 60 \mathrm{~cm}$, con tessere bianche, nere, blu, marroni, ocra, rosse, rosa e verdi. Il campo epigrafico è composto dall'epigrafe:

RUFO DVL (hedera) / CISSIMO (hedera) / CONIVGI (hedera) / SUO (hedera) VIVENT / IVS (hedera) SEPVLCRU / M (hedera) ADORNA / IT (chrismon) (hedera).

La cornice del mosaico è data da una fascia monocroma bianca; il campo è interessato da una figura umana, interpretabile come la figura di un orante. L'uomo infatti, in posizione stante, ha entrambe le braccia alzate, con i palmi delle mani rivolte verso lo spettatore. Veste una dalmatica, e nella zona 


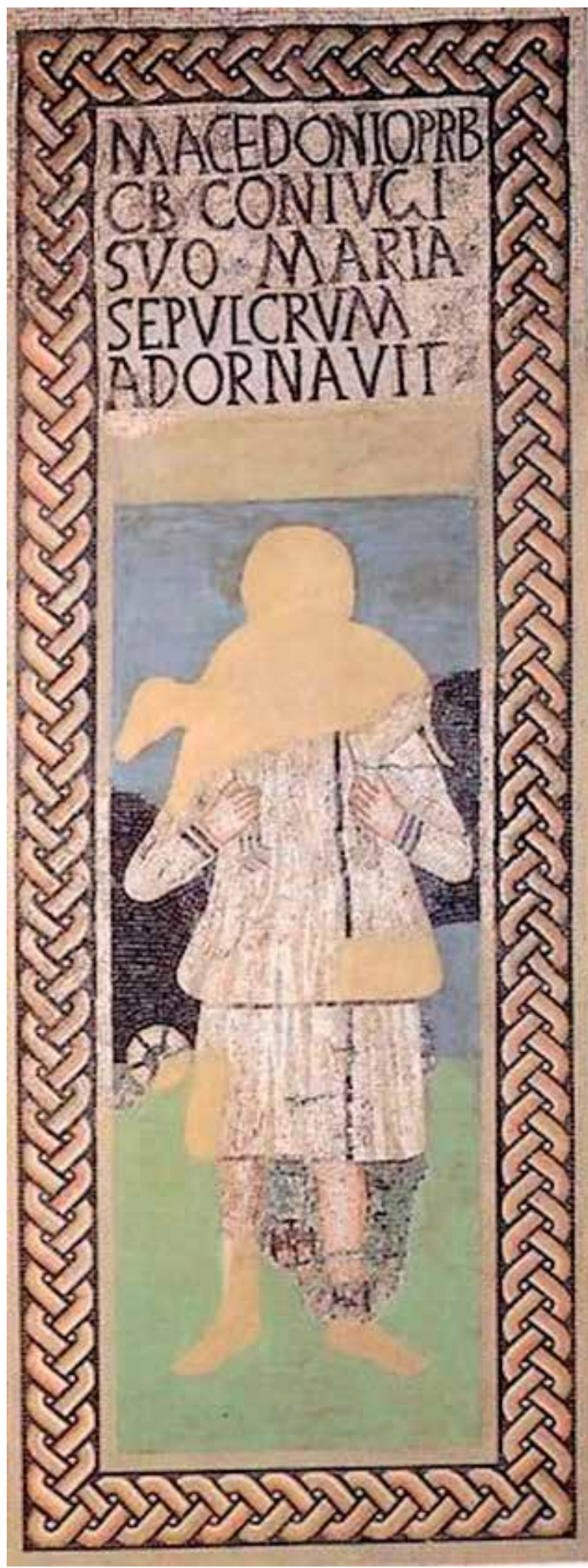

Fig. 5 Mosaico n. 4 di Macedonio, Coscojuela de Fantova, Huesca, Museo Diocesano de Huesca (foto dell'Autore) bassa della veste si scorgono due medaglioni, probabilmente cuciti nella dalmatica stessa. La figura è circondata da delle forme di pane, alle quali si aggiungono, probabilmente, dei pesci. La parte bassa è decorata da due dischi solari e da due colombe. In eccellente stato di conservazione. Databile alla seconda metà del IV secolo (Del Arco, 1921; De Palol, 1967, 332; Schlunk y Hauschild, 1978, 22-23; Lostal,1980, 41; Dominguez, Magallón y Casado, 1984, 77-78; Fernández Galliano, 1987, n. 107; Beltrán Lloris, 1987, n. 9; Blázquez Martínez, 1993, 476479; Gómez Pallarès y Mayer, 1996, 59-60; De Palol, 1999, 303, Gómez Pallarès, 2002, 36-37; Beltrán Lloris, 2004, 51-54; Blázquez Martínez, 2014, 253).

6.

Sempre da Coscojuela de Fantova proviene questo mosaico, sebbene sia rimasta solo una fotografia e allo stato attuale della ricerca sembrerebbe andato perduto. Dalla fotografia non possiamo specificare le dimensioni e la cromia, sebbene possiamo ipotizzare che fossero, simili ai mosaici nn. 4-5. Possiamo però riportare l'iscrizione:

\section{SERGIA [---] / DULCI [---] / MO F [---] / MERE [---] SEPVLC / RUM AD [---] AVER[---].}

Abbiamo anche in questo caso la rappresentazione del defunto nell'iconografia dell'orante, con le braccia stese e i palmi delle mani aperte, con una tunica ampia e circondato da motivi floreali, o verosimilmente da forme di pane come il mosaico n. 5. Anche per questa copertura musiva si protende a una datazione intorno alla seconda metà del IV secolo (Del Arco, 1921; De Palol, 1967, 333; Schlunk y Hauschild, 1978, 22-23; Lostal, 1980, 41; Dominguez, Magallón y Casado, 1984, 72; Fernández Galliano, 1987, n. 108; Gómez Pallarès y Mayer, 1996, 60-61; De Palol, 1999, 303; Beltrán Lloris, 2004, 54-55; Gómez Pallarès, 2002, 37-38).

7.

Il mosaico fu rinvenuto nella Necropoli de Francolí nell'antica Tarraco e oggi è conservato presso il Museo Nazionale Archeologico di Tarragona, con numero d'inventario 2651 (fig. 7). Misura 228 x $82 \mathrm{~cm}$, con tessere di colore bianco, nero, arancione, rosso, blu, verde, ocra e grigio. La cornice è resa con una treccia a doppio capo, nella parte alta del mosaico troviamo l'iscrizione: 


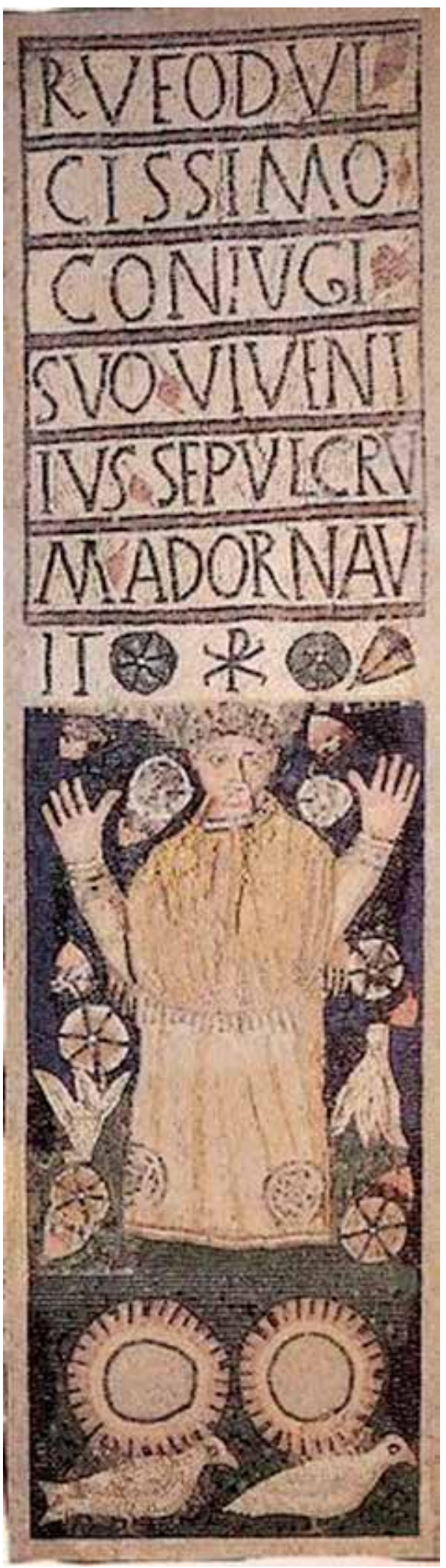

Fig. 6 Mosaico n. 5 di Rufus, Coscojueba de Fantova, Huesca, Museo Diocesano de Huesca (foto dell'Autore)
OPTIME MAGNARUM [---] / CUI MAXIMA RERU[M---] / DIVINAS CAELI QUAS PROMIS+++ / ARCES ECCE DEDIT SANCTA CRHISTI / IN SEDE QUIESCIS[...].

Si tratta di una testimonianza straordinaria poiché l'epitaffio è composto in esametri dattilici. Si noti la "svista" in Crhisti anziché Christi. Sotto l'iscrizione troviamo la raffigurazione di un uomo barbuto, che veste una larga toga trabeata piegata sull'avanbraccio sinistro, la mano destra mostra due dita alzate e la mano sinistra, che spunta dalle pieghe della toga, tiene un volumen. La composizione è arricchita da fiori che circondano il personaggio. La parte figurata, sebbene di straordinaria preziosità nella sua realizzazione, manca di simmetria: si notino infatti che gli adorni floreali sembrano aggiunti in seguito, e non circondano per interno la figura dell'uomo, come se fossero in realtà dei semplici riempitivi. Sebbene lacunoso versa in ottimo stato di conservazione, databile al primo venticinquennio del V secolo (Del Amo, 1979, 106,108; Gómez Pallarès y Mayer, 1996, 74-75; De Palol, 1999, 303; Ribera i Lacomba, 2000, 268; Gómez Pallarès, 2002, 67-68; Blázquez Martínez, 2014, 254-255)

Il gruppo tripartito è formato dal mosaico:

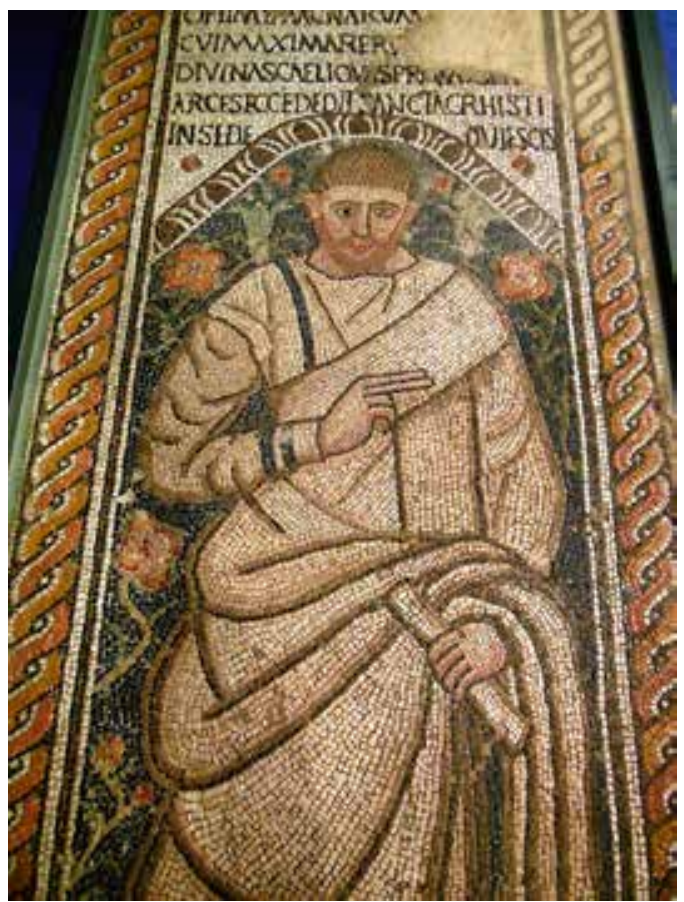

Fig. 7 Mosaico n. 7 rinvenuto presso la Necropoli de Francolí, Tarragona, Museo Nacional Arqueológico de Tarragona, n. Inv. 2651 (foto dell'Autore) 
8.

Il mosaico fu rinvenuto nella Necropoli di Santa Margarita, nell'antica Emporiae, oggi conservato preso il Museo Monografico d'Empurie (fig. 8). I colori impiegati sono: bianco, nero, ocra e rosso. La cornice è composta da tre fasce monocrome, di colori alternati, bianco e nero, e da una treccia a doppio capo, il campo è inquadrato da un'ulteriore cornice dentellata, che racchiude una tabula, con ai lati due quadrati: il quadrato di sinistra ha una decorazione basata su quadrati concentrici, il quadrato destro possiede una ruota dentata con all'interno due cerchi concentrici. L'epigrafe si trova disposta al centro del campo epigrafico, su tre linee rimarcate da tre fasce rosse:

[HIC -circ. 9- C] ORPUS QUIESCET / GAVDET SPIRITVS QVI IN CRISTO LETA / TVR PLVS MINVS VIXIT ANIS LX.

Il mosaico è lacunoso, ma versa in discreto stato di conservazione. Databile al primo quarto del V secolo (Mayer, 2008, 145-150; Tremolada , Castanyer, Santos y Aquilué, 2012, 331-357).

Il gruppo quadripartito è composto dai mosaici:

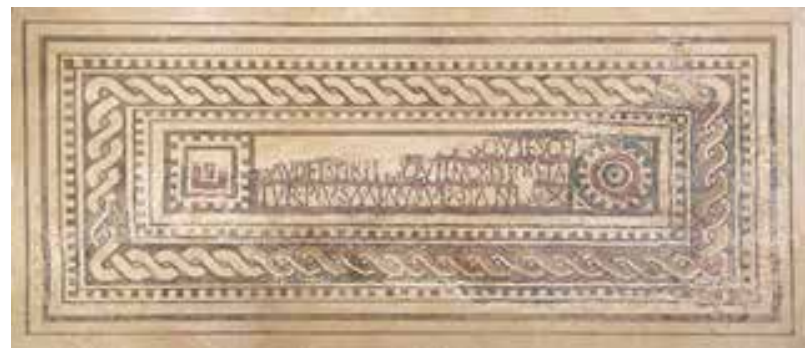

Fig. 8 Mosaico n. 8rinvenuto nella Necropoli di Santa Margarita, Emporiae, Museo Monográfico d'Empurie (foto dell'Autore)

9.

Il mosaico venne rinvenuto ad Alfaro, in provincia di Logroño, insieme a resti di una necropoli paleocristiana. Ora si trova presso il Museo Archeologico Nazionale di Madrid con numero d'inventario 1932/56 (fig. 9). Misura 258 x $92 \mathrm{~cm}$ e possiede tessere in bianco, nero, turchese, granato, ocra, rosso e verde. L'iscrizione recita:

URSIC / INUS / INPACE / DO / PER N / OM / EN / DEI / RECESIT / ANN / XXXXVII REMI / SIT FILIAM AN / N VIII UXOR FEC / IT MELETE.

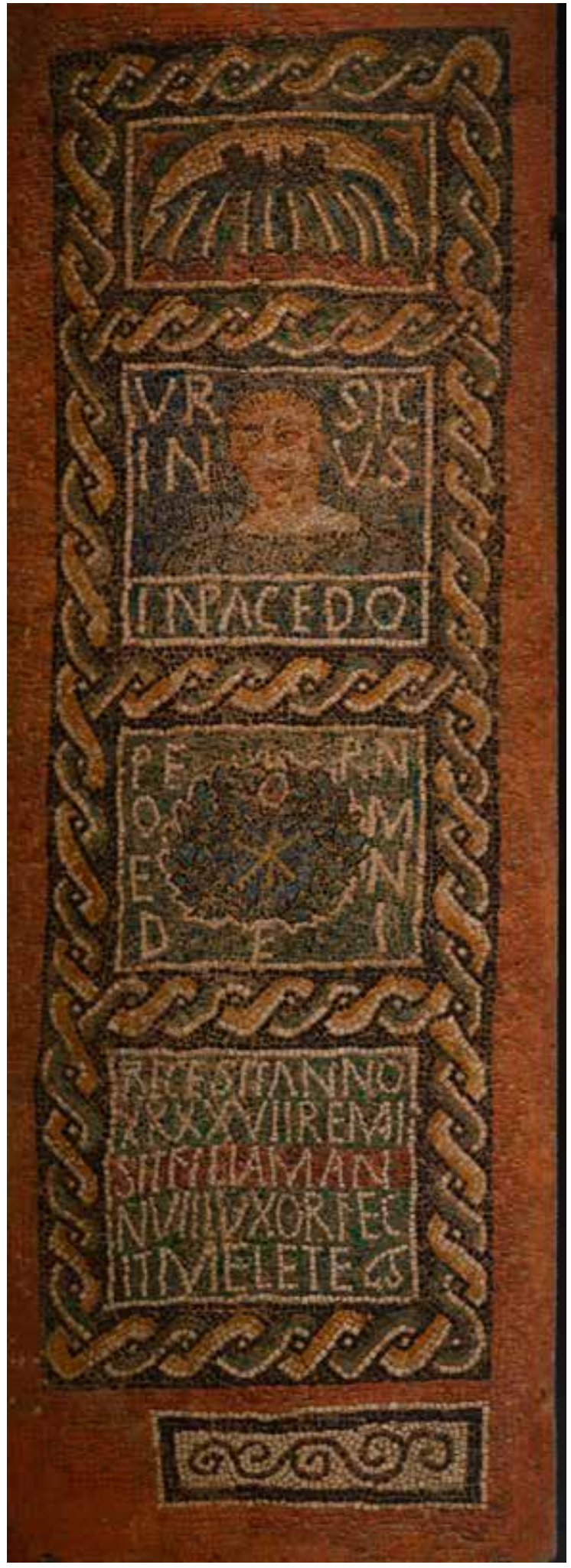

Fig. 9 Mosaico n. 9 di Ursicinus, Alfaro (Logroño), Museo Arqueológico Nacional di Madrid, n. Inv. 1932/56 (foto dell'Autore) 
La spessa treccia a doppio capo, che funge da cornice, racchiude a sua volta i quattro riquadri di questo mosaico. Nella parte alta del mosaico troviamo una venera; nel riquadro successivo abbiamo il ritratto del defunto, sicuramente non realistico perché reso con tratti giovanili, nonostante al momento della morte avesse 47 anni; il terzo riquadro contiene una corona d'alloro con al centro un chrismon; il quarto riquadro invece è interamente occupato dall'ultima parte dell'iscrizione. Si sa che nella sepoltura furono trovata tre monete riferibili a Costanzo II (datata dal 353 alla fine del principato di Costanzo II, 361), sebbene mai si sia saputo che tipo di monete fossero. Il mosaico è in ottimo stato di conservazione. In virtù del ritrovamento numismatico, e prendendo le dovute cautele dal voler dare troppa importanza alle tre monete, che solo possono fornirci un terminus post quem, con accortezza il mosaico è da datarsi tra il 360 e il 390 (De Palol, 1967, 335;Vives, 1969, 258; Elorza, Albertos y Gonzáles, 1980, n. 65; Espinosa, 1986, n. 2; Pérez, 1986, 152-153; Fernández Galliano, 1987, n. 213; Blázquez Martínez, Lopez Monteagudo, Neira Jiménez y San Nicolas Pedraz, 1989, n. 12; Gómez Pallarès y Mayer, 1996, 65-67; Gómez Pallarès, 2002, 59-60; Blázquez Martínez, 2014, 255-256).

10.

Questo mosaico fu ritrovato a Tarraco nella Necropoli de Francolí e oggi conservato presso il Museo Nazionale Archeologico di Tarragona con numero d'inventario 2650 (fig. 10). Misura 182 × 69 $\mathrm{cm}$, con tessere di colore bianco, nero, blu, rosso, ocra, verde, grigio e giallo. Presenta un'iscrizione:

\section{AMPELI/ IN PACE/ REQUIES/CAS.}

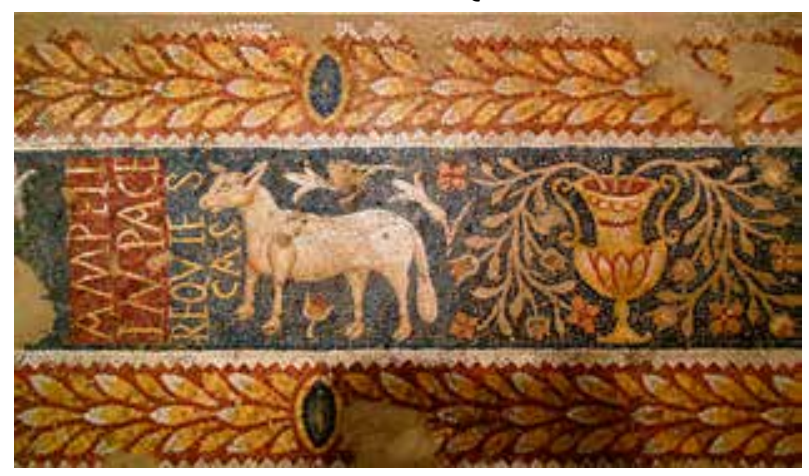

Fig. 10 Mosaico n. 10 di Ampelius, ritrovato nella Necropoli de Francolí, Tarragona, Museo Nacional Arqueológico de Tarragona, n. Inv. 2660 (foto dell'Autore)
La cornice del mosaico è resa da una ghirlanda d'alloro, e la suddivisione del campo musivo non è data da nessun riquadro. Nella parte bassa (per chi leggeva l'iscrizione) ritroviamo un cratere a calice, dal quale spuntano viticci, successivamente, quasi al centro del campo, abbiamo un animale, verosimilmente un agnello, subito dopo è presente l'iscrizione, che a discapito della resa stilistica dell'intero mosaico, sembra opera di una mano inesperta, infine nella parte alta della copertura si ha una grande lacuna che rende illeggibile la figura. Sebbene il mosaico presenti una pesante lacuna, versa in ottimo stato di conservazione. Databile al primo quarto del V secolo (Del Amo, 1979, 106,109; Gómez Pallarès y Mayer, 1996, 75-76; De Palol, 1999, 304; Gómez Pallarès, 2002, 68-69).

L'ultimo gruppo, infine, è rappresentato da mosaici che il loro stato di conservazione non sono inquadrabili in nessuna delle categorie citate per via della loro frammentarietà, e sono:

11.

Mosaico rinvenuto presso la Chiesa di Santa Maria di Terrassa, in situ (fig. 11). Misura 70 × 60 $\mathrm{cm}$, con tessere bianche, nere, blu, grigie, rosse e verdi. Non presenta nessuna iscrizione. Il bordo è reso da fasce monocrome, bianche e nere, alternate, e il campo musivo è decorato da un pavone sulla parte sinistra e un cratere a calice sulla destra. Ampiamente lacunoso ma in buono stato di conservazione, Databile alla fine del IV secolo (Barral, 1978, n. 143; Gómez Pallarès y Mayer, 1996, 56).



Fig. 11 Mosaico n. 11, rinvenuto presso la Chiesa di Santa Maria d Terrassa (foto dell'Autore) 
12.

Il mosaico fu rinvenuto a Coscojuela de Fantova, e oggi conservato al Museo de Huesca. Del mosaico restano 4 frammenti: a) $50 \times 20 \mathrm{~cm}$; b) $7,5 \times 7,5 \mathrm{~cm}$; c) $12,5 \times 12 \mathrm{~cm}$; d) $12,5 \times 12 \mathrm{~cm}$. Le tessere hanno color bianco, nero, blu e verde. L'iscrizione, di difficile lettura, può esser resa con:

[SEPULC]RUM [ca- 1-] + + + + / ETER[---]/ IN [PACE VI]XIT / ANNIS [QU?] A<D $>$ RA[G]IN / TA PL[US] MEN[US] ADOR / NAVIT.

Le enormi lacune non permettono di leggere perfettamente la decorazione, che comunque era resa da una cornice con treccia a doppio capo e probabilmente una colomba. Sarebbe ipotizzabile che anche questa copertura tombale musiva fosse simile alle altre (qui chiamati mosaico 4-5-6), dunque decorato con una figura umana, del Buon Pastore (n. 4) o dell'orante (nn. 5-6). Enormemente lacunoso, in pessimo stato di conservazione. Databile alla seconda metà del IV secolo (Del Arco, 1921; De Palol, 1967, 333; Schlunk y Hauschild, 1978, 22-23; Lostal, 1980, 41; Domínguez, Magallón y Casado, 1984, 72; Fernández Galliano, 1987, n. 108; Gómez Pallarès y Mayer, 1996, 61-63; Beltrán Lloris, 2004, 54-56; Gómez Pallarès, 2002, 38-39).

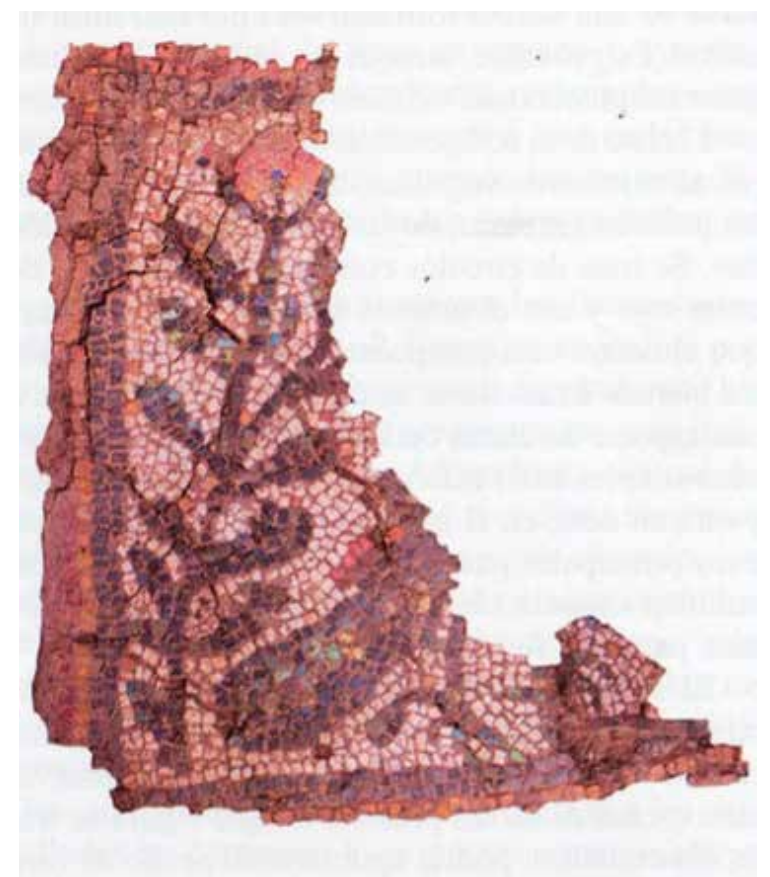

Fig. 12 Mosaico n. 14, ritrovato presso la Calle Mosén Pedro Dosser, Tomba III, Zaragoza (da Galve - Blanco - Cebolla 2005)
13.

Mosaico rinvenuto a Tarraco nella Necropoli de Francolí, oggi conservato presso il Museo Nazionale Archeologico di Tarragona, con numero d'inventario 2649. Misura 208 x $79 \mathrm{~cm}$, con tessere di color bianco, nero, verde, grigio, blu, giallo e rosso. La trascrizione dell'iscrizione è:

A chrisom $\Omega /$ O/ I/ IS/ AT/ ce.

Risulta incorniciato da una ghirlanda d'alloro, con enormi lacune nel campo musivo, unico elemento superstite è una corona doppia, inscritta in un quadrato, con al suo interno un chrismon. Da segnalare che l'alpha è resa nella sua forma latina e non greca. Evidenti lacune, nonostante ciò in buono stato di conservazione (De Palol, 1967, 330; Del Amo, 1979, 106, 109; Gómez Pallarès y Mayer, 1996, 76-77; Gómez Pallarès, 2002, 69).

14.

Il mosaico venne ritrovato presso la Calle Mosén Pedro Dosser, sopra la tomba III, a Zaragoza, oggi conservato presso il Museo Diocesano della stessa città (fig. 12). Misura 130 x $62 \mathrm{~cm}$, con tessere bianche, nere, gialle, blu, rosse e verdi. Non presenta alcuna iscrizione. La cornice è resa da una semplice doppia fila di tessere nere, e il campo musivo, molto lacunoso, è decorato da una rosa con lungo stelo e da un pavone. Evidenti lacune, lo stato di conservazione è discreto. Non si hanno elementi utili per dare una datazione precisa, sebbene questa debba collocarsi al IV secolo, datazione resa possibile grazie ai materiali datanti (Galve y Blanco, 2002, 411; Galve, Blanco y Cebolla, 2005; 490- 496; Galve y Mostalac, 2007, 90-91).

15.

Il mosaico venne ritrovato nella stessa zona del n. 14, e copriva la tomba IV. Attualmente conservato presso il Museo Diocesano di Zaragoza (fig. 13). Composto da due frammenti di dimensioni: a) $71 \times 44 \mathrm{~cm}$; b) $40 \times 50 \mathrm{~cm}$, con tessere di colore bianco, nero, giallo, blu, rosso e verde. La cornice è formata da due linee di tessere in colore nero, e il campo musivo è occupato da colombe e da alcuni frutti. Una colomba ha la cresta allargata che termina con un piumaggio ondulato. Molto lacunoso, in discreto stato di conservazione. Per la datazione si rimanda al mosaico n. 14 (Galve y Blanco, 2002, 411; Galve, Blanco y Cebolla, 2"5; 490- 496; Galve y Mostalac, 2007, 90-91). 


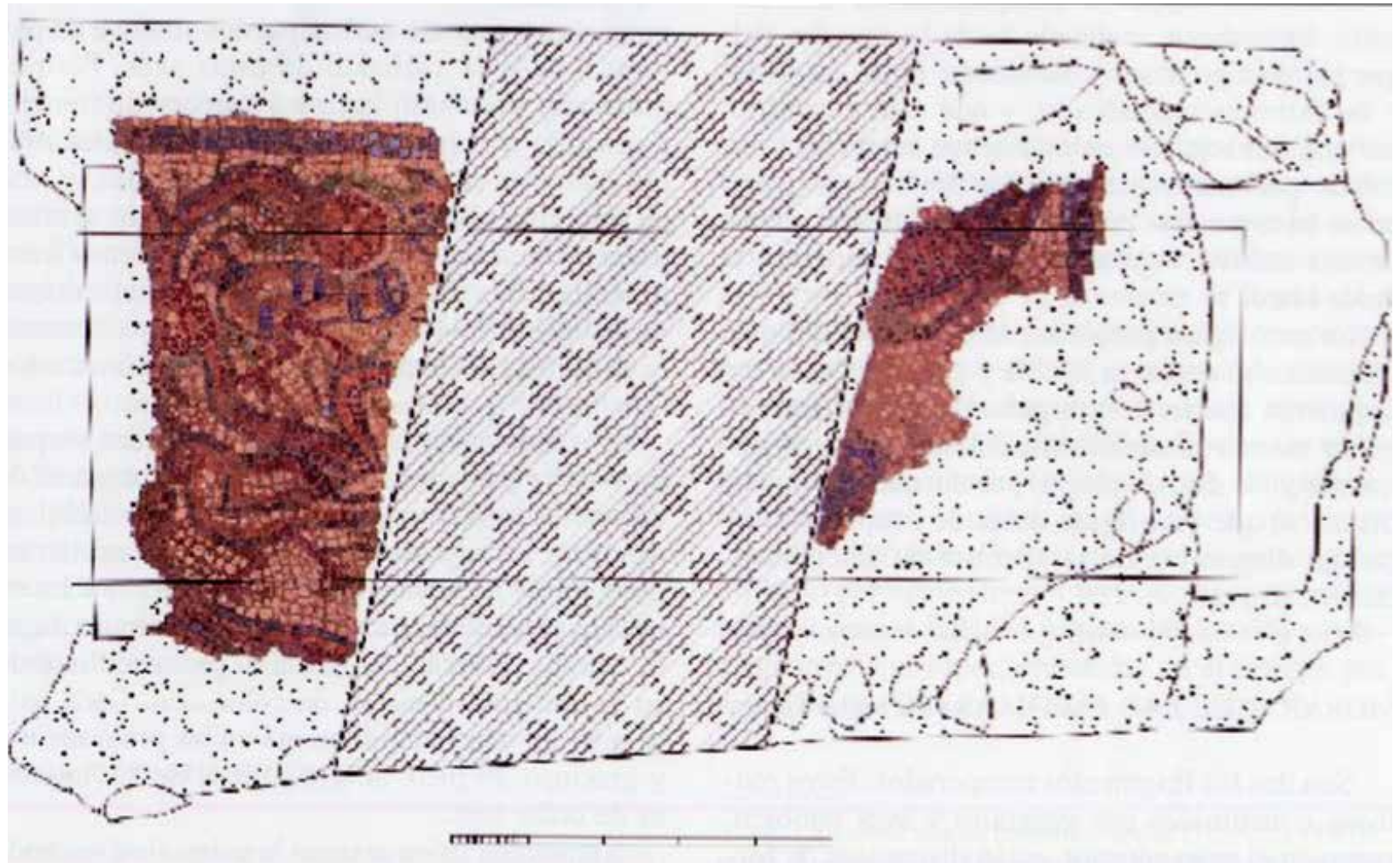

Fig. 13 Mosaico n. 15 presso la Calle Mosén Pedro Dosser, Tomba IV, Zaragoza (da Galve - Blanco - Cebolla 2005)

\section{CONFRONTI}

Iniziando dal primo mosaico analizzato, il n. 1 dobbiamo dire subito che è carente di confronti stringenti, non tanto per la sua tipologia (risulta essere a tutti gli effetti una tipologia ampiamente utilizzata), quanto per la decorazione. Possiamo però ricondurlo a due mosaici di Thagamuta, non ancora datati (Bejaoui, 2003, 306), e a tre mosaici di Sousse della seconda metà del IV secolo (Foucher, 1960, 200-202). Per quanto riguarda i girali vegetali, anche in questo caso son di un tipo abbastanza standardizzato; nella provincia di Granada, nella Villa de Los Vergeles (Marín Diaz, 2011, 176-180) troviamo girali abbastanza simili. Per i mosaici nn. 2, 4, 5, 6, 7, si possono addurre gli stessi confronti. I mosaici nn. 2 e 4 possiedono entrambi una raffigurazione del Buon Pastore, una figura umana in piedi che tiene un agnello per le zampe, posizionato sopra le spalle. Un'iconografia largamente utilizzata nell'arte musiva paleocristiana e nella pittura, che riprende una più antica iconografia, ovvero quella di Orfeo (Jesnick, 1997, 38-44). Esempi, oltre nelle pitture ritrovate nelle Catacombe di Priscilla a Roma (Braconi, 2013, fig. 5), li abbiamo anche ad Aquileia, in mosaici datati tra i secoli IV e V (Bisconti y Braconi, 2012, 231-240). Discorso simile per quanto riguarda l'orante, che lo ritroviamo nei mosaici nn. 5 e 6 , la cui iconografia prende molto in prestito da quella del Buon Pastore. Nel Nord Africa abbiamo due scuole molto attive nella messa in opera di coperture musive tombali recanti l'orante: la prima a Tabarka e la seconda a Sfax. A Tabarka si hanno due botteghe, attive in momenti differenti, una che opera alla fine del IV secolo, l'altra per tutta la prima metà del V secolo (Alexander, 1987, 1-11). A Sfax invece pare esserci solo una bottega, attiva durante la prima metà del V secolo (Yacoub, 2002, 370). Gli esemplari ispanici però sembrerebbero ricondursi alla scuola di Tabarka, soprattutto per l'abbondanza di elementi figurati che ruotano intorno alla figura umana. Il mosaico 8 è della tipologia tripartita più comune nel bacino mediterraneo, anche se è più comune la variante disposta per verticale. A Thagamuta troviamo un esemplare molto simile (Beajoui, 2003, 33), altri esempi sempre di notevole somiglianza a Pupput, della prima metà del V secolo (Baratte y Duval, 1977, 80-82) e specialmente nella Chiesa del Hotel Paradis, del V secolo (Ben Abed Ben Khader y Duval, 1997, 166). 
Il mosaico n. 9 non possiede nessun parallelo all'interno della Penisola Iberica, e precisamente neanche in tutto il Mediterraneo. Sebbene siano presenti altri mosaici quadripartiti, questo mosaico possiede caratteristiche che lo rendono unico. Possiamo dunque ipotizzare che l'artigiano si sia ispirato ad altri modelli, non tratti dall'arte musiva, ma piuttosto da reperti scolpiti. Infatti notevole somiglianza la troviamo nelle stele della Cisalpina (Scarpellini, 1987), ma anche in quelle di Guadalajara (Díaz Díaz, 2014, 370). I confronti per i mosaici nn. 10 e 13 saranno gli stessi; la qualità artistica di questi due pavimenti è notevole, il bordo è riccamente elaborato, molto simile a un mosaico di Timgad (German, 1969, 101), unica differenza è che la ghirlanda dei nostri due mosaici è caricata da ovali e quadrati. Il cratere invece trova stringenti confronti con un mosaico di El Djem (Terry, 1998, 69-70). Sicuramente l'ispirazione per la creazione di questi due mosaici deriva dall'area algerina (Ferdi, 2005). Difficile lettura per il mosaico n. 11, che presenta tessere di grandi dimensioni e un'esecuzione piana. Il corpo del pavone, realizzato in maniera rigida e mediocre, denota poca esperienza dell'artigiano. Un confronto simile si trova in un mosaico databile tra il II e il III secolo a Merida (Blanco Freijeiro, 1978; San Nicolas Pedraz, 20062007, 481; Corrales Álvarez, 2016, 210-244). Imosaicinn.14e15, provengonodallostessocontesto, e verosimilmente si ispirano agli stessi cartoni. In ambito funerario dobbiamo subito segnalare due mosaici molto simili provenienti da Italica databili entro la fine del IV secolo (Gómez Pallarès, 2002, 65-66; Ruiz Prieto, 2013, 92; Blázquez Martínez, 2014, 256-257), soprattutto per la resa anatomica delle colombe e la trattazione degli elementi floreali. Per le colombe e per i fiori confronti stringenti li abbiamo in due mosaici di Hadrumetum della fine del IV secolo (Cintas y Duval, 1958, 242).

\section{LE BOTTEGHE}

Sebbene le coperture musive siano, numericamente parlando, esigue, possiamo cercare di riconoscere alcune botteghe operanti nei secoli IV e V nella Tarraconensis. La prima bottega da prendere in esame è senza ombra di dubbio quella orbitante nella provincia di Huesca, che realizzò i mosaici $4,5,6$. Si tratta probabilmente di una bottega a raggio molto limitato, altamente locale, che durante la fine del IV secolo trae ispirazione dalle citate botteghe di Tabarka, dalle quali rimodella l'iconografia dell'orante. La qualità artistica, soprattutto nella resa della figura umana, è notevolmente superiore rispetto alle botteghe nord africane, fatto che potrebbe essere spiegato in virtù di un'alta specializzazione in quell'iconografia, e soprattutto nella tipologia del mosaico funerario. Non è un caso che la provincia di Huesca abbia restituito il numero più elevato di coperture musive di tutta la Penisola Iberica. La zona della Tarraconensis, che ha restituito i mosaici $2,7,10,11,13$, possedeva una bottega altamente specializzata, per lo meno per i mosaici $2,7,10,13$, probabilmente la bottega più esperta di tutta la penisola. Operante durante il V secolo, dimostra di essere al servizio di facoltosi cittadini (si ricordi la composizione in esametri del mosaico 7 , che denota un alto livello culturale). L'ispirazione è certamente africana, e la sua zona d'azione era la Necropoli de Francolí, anche se visto il livello artistico è pensabile che potesse operare anche nelle domus della stessa città, o più possibile, che gli artigiani provenissero da alcune botteghe che già lavoravano nella città (Schlunk - Hauschild, 1978, $13,78,85,97-98,136,139,145,149-150,152-154$, 172), con maestri proveniente dall'ambiente asiatico (Balil, 1969, 3), e che successivamente si siano discostati, prendendo ispirazioni da ambienti africani e specializzandosi nei mosaici funerari. Per le province di Barcellona e Girona non siamo in grado, allo stato attuale delle ricerche, di individuare con certezza le botteghe. Possiamo però ipotizzare che dovessero trattarsi di botteghe non ispaniche, probabilmente di matrice itinerante con provenienza nord africana, e che lavorarono tra i secoli IV e V. In ultimo, la bottega di Zaragoza, doveva essere anch'essa itinerante. Di chiara ispirazione africana è possibile che abbia operato, in momenti distinti ma vicini tra loro (seconda metà del IV secolo) nelle città di Italica e Zaragoza.

\section{ConCLUSIONI}

Come abbiamo visto le coperture tombali musive non ebbero grande fortuna in territorio ispanico, per quanto la Tarraconensis sia la regione che ci ha restituito il maggior numero di testimonianze. L'ispirazione di questi mosaici è di chiara natura africana, anche se è da evidenziare che la versatilità e l'originalità di talune botteghe locali, abbiano plasmato e rimodellato i vari cartoni provenienti dai territori africani, talvolta creando dei pezzi unici. Non ci deve stupire questo fatto, poiché in tutto il 
territorio dell'Hispania gli artigiani si son sempre distinti per la loro capacità compositiva, nonostante alcuni studiosi affermino che l'arte musiva ispana risenta di pesanti influssi africani (Dundabin, 1987, 251). Anche per il mosaico funerario è stato affermato che siano di qualità bassa e dallo stile floscio (Blázquez Martínez, 2014, 262), mentre in realtà, alcuni mosaici, si distinguono per l'eccellente esecuzione, talvolta di livello maggiore rispetto ai parenti nord africani. Questo fatto è facilmente spiegabile: nel Nord Africa assistiamo a una seriazione della produzione (Raynal, 2005), mentre in Hispania, la minor richiesta permette agli artigiani una maggiore specializzazione, che non si può avere con una produzione continua e in serie. Rimane aperta la questione della poca fortuna di questa tipologia musiva. Il prodotto è di chiara origine nord africana, probabilmente tunisina, vista l'ampiezza del fenomeno in quei territori, e la sua importazione sulla Penisola Iberica è da imputarsi ai massicci commerci che intercorrevano tra i due territori, i quali, in questi secoli oltre a materiale di uso comune, portarono materiali di lusso, come i famosi sarcofagi di Cartagine (Rodá, 1990, 727736). Probabilmente la poca espansione di questo fenomeno può essere ricercato nella poca cristianizzazione, in questi secoli, dell'Hispania. Infatti son pochi i martiri presenti durante i secoli $\mathrm{IV}^{4} \mathrm{e}$ V (Sotomayor, 1979, 66-70), molto ampie son le zone della Spagna che non possiedono neanche un martire. Non è forse un caso che la Tarraconensis, invece, abbia un numero maggiore di martiri, un esempio tra tutti è il martirio durante la persecuzione di Valeriano del vescovo Fructuoso (Sotomayor, 1979, 49-58). Oltretutto il Cristianesimo attecchì maggiormente in territorio urbano (appunto Tarragona), mentre la Hispania era fortemente colpita dal fenomeno della villa rustica, che probabilmente impedi, almeno inizialmente, il diffondersi massiccio della nuova religione.

4 Un caso anomalo è la città di Emerita Augusta, per la quale si possono vedere: Mateos Cruz, 1999; Camacho Macías, 2006; Cordero Ruiz, 2013.
Bibliografia

Alexander, M. A. (1987), "Mosaic Ateliers at Tabarka”, Studies on Art and Archaeology in Honor of Ernst Kitzinger on His Seventy-Fifth Birthday, Dumbarton Oaks Papers, 41, 1-11.

Álvarez, A. y Mayer, M. y Rodà, I. (1987), "Los materiales lapídeos reaprovechados en Catalunya. La ciudad de Barcelona y su entorno", Artistes, Artisans et production artistique au Moyen Age, Paris, 529-558.

Balil, A. (1955), "Una nueva lauda sepulcral de mosaico en el Conuentus Tarraconense," Archivo Español de Arqueología, 91, 142-145.

Balil. A. (1969), "Il mosaico della Medusa di Tarragona”, Latomus, 103, Hommanages à $M$. Renard, 3-12.

Baratte, F. y Duval, N. (1977), Catologue des mosaïques romaines et paléochrétiennes $d u$ Musée du Louvre, Parigi.

Barral, X. (1978), Les mosaïques romaines et médiévales de la Regio Laietana (Barcelona et ses environs), Barcelona.

Bejaoui, F. (2003), "Les mosaïques funeraires de Sufetula (Sbeitla) et de Thagamuta (Kasr El Baroud)", Actes du VIII Colloque International sur l'Histoire et l'Archéologie de l'Afrique $d u$ Nord ( Khanoussi, M., ed), Tunisi, 303-334.

Beltrán Lloris, F. (1987), "Epigrafía y romanización en la provincia de Huesca", Annales, 4, 19-34.

Beltrán Lloris, F. (2004), "Notas sobre las inscripciones latina de Coscojuela de Fantova (Huesca)”, Sylloge epigraphica Barcinonensis, 5, 31-63.

Ben Abed, A. y Duval, N. (1997), "Les mosaïques funéraires d'une église de Pupput (Hammamet- Tunisie): Etudes d'Archéologie chrétienne nord-africaine, XXV", Antiquités Africaines, 33, 165-190.

Bisconti, F. y Braconi, M. (2012), "Il riuso delle immagini in età tardoantica: L'esempio del Buon Pastore dall'Abito Singolare", Antichitì Altoadriaticheci, 74, 229-240.

Blanco Freijeiro, A. (1978), Mosaicos Romanos de Merida, Madrid.

Blázquez Martínez, J. M., López Monteagudo, G., Neira Jiménez, $M^{\mathrm{a}}$ L. y San Nicolás Pedraz, $M^{\mathfrak{a}}$. P. (1989), CME IX, Madrid.

Blázquez Martínez, J. M. (2014), "Mosaicos sepulcrales paleocristianos hispanos con figuras humanas y sus paralelos del África Procunsular”, 
Religiosidad, rituales y prácticas mágicas en los mosaicos romanos (Luz Neira, ed.), Madrid, 253-265.

Braconi, M. (2013), "Il pastore e le belve. Crasi iconografiche e paradossi tematici sulla lastra

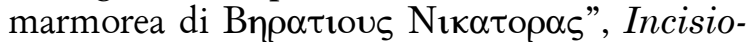
ni Figurate dalla Tardo Antichità. Atti del Convegno di Studi, (Bisconti, F. y Braconi, M., ed.), Città del Vaticano, 58-80.

Cintas, J. y Duval, N. (1958), "L'église du prête Felix dans la région de Kélibia”, Karthago, IX, 179-265.

Corrales Álvarez, A. (2016), La arquitectura doméstica de Augusta Emerita, Madrid.

De Palol, P. (1967), Arqueología Cristiana de la España Romana. Siglos IV-VI, Madrid.

De Palol, P. (1999), Del Romà al Romànic. Història, art $i$ cultura de la Tarraconse mediterrània entre els segles IV $i \mathrm{X}$, Barcelona.

Del Amo, D. (1979), Estudio crítico de la necrópolis paleocristiana de Tarragona, Tarragona.

Del Arco, R. (1919), "Nuevos restos romanos hallados en Coscojuela de Fantova (Huesca)", Boletín de la Real Academia de la Historia 75, 127-142.

Del Arco, R. (1921), Excavaciones en monte Cillas, término de Coscojuela de Fantova (Huesca), Memorias de la Junta Superior de Excavaciones y Antigüedades, Madrid.

Díaz Díaz, T. (2014), "Evolución del retrato funerario: la necesidad de perpetuidad", El mundo de los difuntos: culto, cofradias y tradiciones, San Lorenzo del Escorial, 623-640.

Domínguez, A. , Magallón, M. A. y Casado, M. P. (1984), Carta Arqueológica de España, Huesca. Dunbabin, K. M. D. (1978), The Mosaics of Roman North Africa. Studies of Iconografy and Patronage, Oxford.

Elorza, J. C., Albertos, M. L. y Gonzáles, A. (1980), Inscripciones Romanas en la Rioja, Logroño. Espinosa, U. (1986), Epigrafía romana de la Rioja, Logroño.

Ferdi, S. (2005), Corpus de mosaïques de Chercel, Parigi.

Fernández Galiano, D. (1987), Mosaicos Romanos del convento cesaraugustano, Zaragoza.

Foucher, L. (1960), Guide du musée de Sousse, Tunisi.

Galve, P. y Blanco, A. (2002), "Nuevos datos para la arqueología funeraria de Caesaragusta: las tumbas paleocristianas de la Calle Mosén Pedro Dosset (Vial) Zaragoza”, Saldie, 2, 409-411. Galve, P., Blanco, A. y Cebolla, J. L. (2005), "Edificio de culto paleocristiano en Zaragoza", VI Reunió d'arqueología Cristiana Hispánica, Barcelona, 483-498.

Galve, P. y Mostalac, A. (2007), Las necrópolis, en Zaragoza. Colonia Caesar Augusta, F. Beltrán (ed.), Roma.

Germain, S. (1969), Les mosaïques de Timgad. Étude descriptive et analytique, Parigi.

Godoy Fernández, (1995), Arqueología y liturgia. Iglesias hispánicas (siglos VI al VIII), Barcelona.

Gómez Pallarès, J. y Mayer, M. (1996), “Aproximación a un inventario de los mosaicos funerarios de época paleocristiana de Hispania”, Cahiers des études anciennes, XXXI, 49-96.

Gómez Pallarès, J. (2002), Epigrafía cristiana sobre mosaico de Hispania, Roma. Jesnick, I. J. (1997), The Image of Orpheus in Roman Mosaics, Oxford.

Marín Díaz, P. (2011), "Una aproximación a la musivaria tardoantigua en Iliberis. Los mosaicos de la villa de Los Vergeles (Granada)", Arqueología y Territorio, 8, 173-186.

Mayer, M. (2008), "La nova lauda en mosaic d'Empuries: alguns suggeriments de lectura", en $S y l$ loge epigraphica Barcinonensis , VI, 145-150.

Pérez, Ma J. (1986), Los Vascones, Pamplona.

Puig i Cadafalch, J. (1947), "La cathédrale et le baptistère d'Egara" (Catalogne), Comptes rendus des séances de l'Académie des Inscriptions et Belles-Lettres, 91/3,536-540.

Raynal, D. (2005), Archéologie et histoire de l'Église d'Afrique. Uppenna II. Mosaïque funéraires et mémoire de martyrs, Parigi.

Ribera i Lacomba, A. (2000), Los orígines del cristianismo en Valencia y su entorno, Valencia.

Rodá, I. (1990), "Sarcofagi della bottega di Cartagine a Tarraco", L'Africa romana. Atti del VII Convegno di studio, Sassari, 727-736.

Ruiz Prieto, E. (2013), "Itálica tardoantigua: reflexiones y asignatura pendientes”, Ligustinus, 1, 81-117.

San Nicolás Pedraz, Mª . P. (2006-2007), "Motivos de xenia en los mosaicos romanos de Hispania”, Espacio, Tiempo y Forma, Serie II, Historia Antigua, 19/20, 469-497.

Scarpellini, D. (1987), Stele romane con imagines 
clipleate in Italia, Roma.

Sotomayor, M. (1979), Historia de la Iglesia en España. I. La Iglesia en la España Romana y Visigoda, Madrid.

Schlunk, H. y Hauschild, Th. (1978), Die Denkmäler der frühchristlichen un westgotischen Zeit, Mainz.

Terry, J. H. (1998), Christian Tomb Mosaics of Late Roman, Vandalic and Byzantine Byzacena, Tesi di dottorato, University of Missouri-Columbia.

Tremoleda, J. y Castanyer, P. y Santos, M. y Aquilué, X. (2012), "Las necrópolis tardoantigues $i$ altmedieval d'Empúries (L'Escala, Alt Empordà)", Arqueología funeraria al nord-est peninsular (segles VI- XII), (N. Molist i G. Ripoll, ed.), Barcelona, 331-357.

Yacoub, M. (2002), Splendeur des Mosaïques de Tunisie, Tunisi.

Vives, J. (1969), Inscripciones Cristianas de la España Romana y Visigoda, Barcelona. 
